\title{
A NOTE ON THE ADJOINT OF A PERTURBED OPERATOR
}

\author{
BY R. W. BEALS ${ }^{1}$
}

Communicated by Felix Browder, October 18, 1963

Let $X$ and $Y$ be Banach spaces. By an operator from $X$ to $Y$ we mean a linear operator with domain $D(T) \subseteq X$ and range $R(T) \subseteq Y$. An operator $T$ from $X$ to $Y$ is said to be a Fredholm operator if $T$ is closed, the null space $N(T)$ has finite dimension, and the range $R(T)$ is closed and has finite codimension in $Y$. We denote by $\Phi(X, Y)$ the set of all Fredholm operators from $X$ to $Y$. If $T \in \Phi(X, Y)$, the index of $T$ is defined to be

$$
\operatorname{ind}(T)=\operatorname{dim}(N(T))-\operatorname{codim}(R(T)) .
$$

Suppose $T \in \Phi(X, Y)$. Since $T$ is closed, the graph $G(T)$ is a closed subspace of $X \times Y$. An operator $C$ from $X$ to $Y$ is said to be $T$-compact if $C$ is closable, $D(C) \supseteq D(T)$, and the mapping $(x, T x) \rightarrow C x$ is compact as an operator from $G(T)$ into $Y$.

The following results are well known:

(1) If $T \in \Phi(X, Y)$ and $C$ is $T$-compact, then $T+C \in \Phi(X, Y)$ and $\operatorname{ind}(T+C)=\operatorname{ind}(T)$. (See $[2$, Theorem 2.6].)

(2) If $T$ is a closed operator from $X$ to $Y$ and $D(T)$ is dense in $X$, then $T$ is in $\Phi(X, Y)$ if and only if the adjoint operator $T^{*}$ is in $\Phi\left(X^{*}, Y^{*}\right)$. If so, then $\operatorname{ind}\left(T^{*}\right)=-\operatorname{ind}(T)$. (For by $[1$, Theorem A], $R(T)$ is closed if and only if $R\left(T^{*}\right)$ is closed. If so, then $\operatorname{dim}\left(N\left(T^{*}\right)\right)$ $=\operatorname{codim}(R(T))$ and $\operatorname{codim}\left(R\left(T^{*}\right)\right)=\operatorname{dim}(N(T))$.)

Lemma. Suppose $S \in \Phi(X, Y), T \in \Phi(X, Y)$, and $S \subseteq T$. Then $\operatorname{ind}(S) \leqq \operatorname{ind}(T)$, and equality holds if and only if $S=T$.

Proof. If $S \subseteq T$, then $N(S) \subseteq N(T)$ and $R(S) \subseteq R(T)$, so the inequality is obvious. Equality implies $N(S)=N(T)$ and $R(S)=R(T)$, so $S=T$.

Proposition. Suppose $T \in \Phi(X, Y)$ and $D(T)$ is dense in $X$. If $C$ is an operator from $X$ to $Y$ such that $C$ is $T$-compact and $C^{*}$ is $T^{*}$-compact, then $T^{*}+C^{*}=(T+C)^{*}$.

Proof. From (1) and (2) it follows that $T^{*}+C^{*} \in \Phi\left(X^{*}, Y^{*}\right)$ and $(T+C)^{*} \in \Phi\left(X^{*}, Y^{*}\right)$. Furthermore

$$
\begin{aligned}
\operatorname{ind}\left(T^{*}+C^{*}\right)=\operatorname{ind}\left(T^{*}\right) & =-\operatorname{ind}(T) \\
& =-\operatorname{ind}(T+C)=\operatorname{ind}\left((T+C)^{*}\right) .
\end{aligned}
$$

${ }^{1}$ National Science Foundation Graduate Fellow. 
It is obvious that $T^{*}+C^{*} \subseteq(T+C)^{*}$, and equality follows from the lemma.

Remark. If $C$ is $T$-compact, $C^{*}$ need not be $T^{*}$-compact. In fact it can happen that $D\left(C^{*}\right) \cap D\left(T^{*}\right)=(0)$, and therefore $(T+C)^{*}$ $\neq T^{*}+C^{*}$, even when $T$ is the inverse of a positive definite compact Hermitian operator in a Hilbert space.

\section{REFERENCES}

1. F. E. Browder, Functional analysis and partial differential equations. II, Math. Ann. 145 (1962), 81-226.

2. I. C. Gohberg and M. G. Krein, The basic propositions on defect numbers, root numbers, and indices of linear operators, Uspehi Mat. Nauk 12 (1957), 43-118; English transl., Amer. Math. Soc. Transl. (2) 13 (1960), 185-264.

YALE UNIVERSITY 